\title{
Down-regulated Expression Of CDK5RAP3 and UFM1 Suggests A Bad Prognosis In Gastric Cancer Patients Running title: CDK5RAP3 and UFM1 in gastric cancer
}

Mi Lin

Fujian Medical University Union Hospital

Ning-Zi Lian

Fujian Obstetrics and Gynecology Hospital

Long-Long Cao

Fujian Medical University Union Hospital

Changhwan Yoon

Memorial Sloan Kettering Cancer Center

Chang-Ming Huang

Fujian Medical University Union Hospital

Chao-Hui Zheng

Fujian Medical University Union Hospital

Ping Li

Fujian Medical University Union Hospital

Jian-Wei Xie

Fujian Medical University Union Hospital

Jia-Bin Wang

Fujian Medical University Union Hospital

Jun Lu

Fujian Medical University Union Hospital

Qi-Yue Chen

Fujian Medical University Union Hospital

Ya-Han Li

Fujian Medical University

Zhu-Huai Peng

Fujian Medical University

Xiao-Yu Zhang

Fujian Medical University

Yi-Xian Mei

Fujian Medical University 
Jian-Xian Lin ( $\square$ linjian379@fjmu.edu.cn )

Fujian Medical University Union Hospital

\section{Research Article}

Keywords: Gastric adenocarcinoma, CDK5RAP3, UFM1, AKT pathway, Prognosis

Posted Date: March 7th, 2022

DOI: https://doi.org/10.21203/rs.3.rs-1340611/v1

License: (c) (i) This work is licensed under a Creative Commons Attribution 4.0 International License.

Read Full License 


\section{Abstract}

Purpose: The relationship between the CDK5RAP3 and UFM1 expression and the prolonged outcomes of patients who underwent gastric cancer (GC) surgery was investigated. Methods: Single-sample gene set enrichment analysis (SSGSEA), unsupervised clustering and other methods were used to verify the relationship between CDK5RAP3 and UFM1 in GC through public databases. Additionally, CDK5RAP3 and UFM1 expression in cancerous and paracancerous tissues of GC was analysed in the context of patient prognosis. Results: CDK5RAP3 and UFM1 expression was downregulated synchronously, the interaction was existed between the two proteins, and UFM1 and CDK5RAP3 expression was found to be inversely associated to AKT pathway activation. Prognostic analysis showed that the prognosis is poorer for low CDK5RAP3 and UFM1 patients, than for high CDK5RAP3 and/or UFM1 $(\mathrm{p}<0.001)$ patients, and this expression pattern was an independent predictor for overall survival of GC. Coexpression of CDK5RAP3 and UFM1 combined with TNM staging can improve the accuracy of prognosis prediction for patients ( $p$ $<0.001$ ). Conclusions: It is confirmed in our findings that a combination of CDK5RAP3 and UFM1 can produce a more precise prediction model for GC patients' survival.

\section{Introduction}

As a well-known malignant tumor, gastric cancer leads to high lethality of patients worldwide, which makes it rolling as a leading cause to death. The latest epidemiological survey showed that gastric cancer ranks fifth and third global incidence and mortality rates, respectively, among malignant tumours. Globally, more than one million new gastric cancer cases are diagnosed every year, and approximately 800,000 people die of gastric cancer [1]. At diagnosis and therapy, majority of patients are already at an advanced stage because of low specificity of early gastric cancer symptoms. Advanced gastric cancer patients have an unfavourable prognosis, with just around a 15\% 5-year survival rate. [2]. Accurate prognostic assessment helps to formulate reasonable treatment plans and follow-up plans. The TNM staging system is the foremost predictor for gastric cancer prognosis. However, even with the same TNM stage, the prognosis of patients is not the same. In 2014, data from the Gastric Cancer Genome Atlas Research Network confirmed the molecular heterogeneity of gastric cancer [3]. Therefore, the prognostic evaluation of the biological potential of gastric tumours has attracted increased attention. It has vital theoretical and clinical significance for the prognostic evaluation of gastric cancer to explore the molecular markers for early identification of gastric cancer and the important role of molecular targeted therapy.

CDK5RAP3, known as C53, is an activation binding protein of cyclin-dependent kinase 5; it contains 506 amino acid residues and has a zinc-leucine zinc finger structure [4]. CDK5RAP3 plays a key role in the formation and evolution of various malignant tumours [5]. In our previous researches, we found that CDK5RAP3 can inhibit the phosphorylation of AKT in gastric cancer [6], thereby inhibiting the GSK-3 $\beta$ mediated phosphorylation, degrading $\beta$-catenin and acting as a tumour suppressor in the occurrence and progression of gastric cancer [7]. 
UFM1, a amall ubiquitin protein that contains 85 amino acids, was first discovered by Komatsu in 2004. UFM1 is first activated by UBA5 and is then converted into UFC1 and UFL1. UFL1 recognizes and helps UFM1 to bind the target protein. Finally, UFM1 processes and modifies the target protein to perform its biological vital activities. UFM1 and its modification system participate in different pathophysiological and biological processes, including the cell cycle, fatty acid $\beta$ oxidation, cell survival, and hypoxia tolerance [8-10]. Research has demonstrated that the development of breast cancer involves UFM1 [11]. In previous studies, we found that UFM1 can also negatively regulate PI3K/AKT signalling by increasing the ubiquitination of PDK1 to inhibit the invasion and metastasis of gastric cancer [12].

The Akt-related signal transduction pathway is a complex signalling network mediated by growth factor receptors (GFRs) [13]. Activation of this pathway suppresses cell apoptosis triggered by different stimuli, increases progression and proliferation of the cell cycle, participates in the neovascularization, plays an important role in the formation of tumours, and participates in invasion and metastasis of tumours [1416]. Thereby, we considered that CDK5RAP3 and UFM1 may play a coordinated role in inhibiting the gastric cancer invasion and metastasis. Although some studies have suggested that UFM1 binds to CDK5RAP3, the expression of the two proteins and their effects on the prolonged survival in gastric cancer have not been documented yet.

Therefore, we investigated the correlation between UFM1 and CDK5RAP3 expression and the prognosis of gastric cancer using public databases. We also detected the expression of the two indicators in 215 gastric cancer tissue samples using IHC, Western blotting and qPCR. To improve the accuracy of judging prognosis in gastric cancer, the relationship between expression of these two proteins and relevant clinical and pathological characteristics, as well as long-term survival in patients was analysed.

\section{Materials And Methods}

\section{Gastric cancer data sets}

We searched the published gastric cancer gene expression database systematically, including those with complete clinical information and excluding those with no survival information. Finally, we gathered The Cancer Genome Atlas Stomach Adenocarcinoma (TCGA-STAD) cohorts and 17 Gene Expression Omnibus (GEO) cohorts of samples from patients with GC for this study (GSE54129, GSE65801, GSE35809, GSE51105, GSE13861, GSE27342, GSE29272, GSE63089, GSE19826, GSE79973, GSE13911, GSE51575, GSE118916, GSE122401, GSE130823, GSE15459, GSE66229) and the TCPA database for analysis. The original data were collected and downloaded from GEO (http://www.ncbi.nlm.nih.gov/geo/), TCGA (https://portal.gdc.cancer.gov/), and TCPA (https://www.tcpaportal.org/tcpa).

\section{Tissue specimens}

The tissues in this study were selected from gastric adenocarcinoma tissue specimens of 215 patients undergoing radical gastrectomy for gastric cancer in our center from January 2013 to December 2014. All patients were newly diagnosed and before surgery they had not received chemotherapy or radiation 
treatment. The patients were pathologically confirmed to have gastric adenocarcinoma after surgery with comprehensive clinicopathological information. The data were analyzed retrospectively. This study was approved by the Fujian Medical University Union Hospital Ethics Committee and written permission was obtained from every relevant patient.

\section{Single-sample gene set enrichment analysis (ssGSEA)}

We obtained 3 GFR gene sets (KRAS_SIGNALING_UP and AKT_UP. V1_DN and MTOR_UP. V1_DN) from C6 (oncogenic gene sets) of MSigDB (https://www.gsea-msigdb.org/). Using the R software package "GSVA" (gene set variation analysis for microarray and RNA-seq data), we scored each sample in the TCGA cohort by ssGSEA (method = "ssgsea", ssgsea.norm = TRUE, verbose = TRUE).

\section{Unsupervised clustering}

Unsupervised clustering methods (K-means) were used to classify the TCGA cohort into different clusters based on the enrichment of GFR pathways. The clustering factors were the ssGSEA scores of the three GFR gene sets. These scores were first converted to z scores to improve the accuracy of clustering. We determined the final number of clusters according to the algorithm provided by the R software package "NbClust". Finally, the TCGA queue was accurately divided into 3 clusters defined as Cluster A, Cluster B, and Cluster C.

\section{GSEA}

We performed GSEA on the TCGA and GEO datasets (GSE54129, GSE65801, GSE35809, and GSE51105). First, we used the mean \pm standard deviation (SD) of the CDK5RAP3 expression value as the cut-off point to divide each data set into three groups: the group of high, moderates and low. Next, we compared the high and low expression group to obtain differentially expressed genes. Additionally, the R package "clusterProfiler" (v3.12.0)0 (https://guangchuangyu.github.io/software/clusterProfiler) was applied to perform GSEA on these differential genes. MSigDB provided us with all of the hallmark and oncogenic gene sets (https://www.gsea-msigdb.org/).

\section{Immunohistochemistry}

Tumour specimens containing enough formalin-fixed and embedded by paraffin were sliced into 4- $\mu \mathrm{m}$ serial sections and mounted for immunohistochemical analysis on silane-coated glass slides. The sections were dewaxed, rehydrated, antigen repaired, blocked and then incubated with appropriate antibodies. The rabbit anti-human CDK5RAP3 (ab24189; 1:200; Abcam) or UFM1 (ab109305; 1:200; Abcam) antibody was used as the first antibody.

\section{Immunohistochemical score}

Two experienced pathologists independently assessed IHC-stained tissue slices and scored them based on the intensity of cell staining and the positive ratio of the stained tumour cells. The proportion and intensity of CDK5RAP3-positive and UFM1-positive cells in random selection visual areas were evaluated 
to indicate the protein expression level. The following were the staining score standards for CDK5RAP3 and UFM1: no staining was indicated by a score of 0 ; the light yellow was defined as mild staining with a score of 1 ; the yellowish brown was defined as moderate staining with a score of 2 ; the brown was defined as significant staining with a score of 3 . The following were the proportional score standards for stained tumor cells: when less than or equal to 5 percent cells were positive, the score was 0 ; when the positive cells were range from 6 to 25 percent, the score was 1 ; when the positive cells were range from 26 to 50 percent, the score was 2; when the positive cells were greater than or equal to 50 percent, the score was 3. (Figure S1). The final score ranging from 0 to 9 for the expression of CDK5RAP3 and UFM1, was obtained by multiplying the staining score and proportional score. The low-expression group was defined as patients having a final score $<4$. The high-expression group included the remaining patients.

\section{Western blotting}

The following antibodies were used by Western blots: CDK5RAP3 (ab24189; 1:1000 dilution; Abcam, Cambridge, MA, USA), UFM1 (ab109305; 1:1000 dilution; Abcam, Cambridge, MA, USA), p-AKT (serine 473) (ab81283, 1:1000 dilution; Abcam, Cambridge, MA, USA) and GAPDH (\#5174; 1:2000 dilution; Cell Signaling Technology).

\section{Total RNA extraction and qPCR}

Total RNA from gastric cancer and paracancerous tissues was extracted using Invitrogen's TRIzol kit according to the manufacturer's instructions and used to obtain cDNA using Takara's reverse transcription system. The copy numbers of GAPDH, CDK5RAP3 and UFM1 were detected using qPCR. The following were the detailed primer sequences:

CDK5RAP3 Forward primer: 5'-GCTGGTGGACAGAAGGCACT-3'

Reverse primer: 5'-TGTCCTGGATGGCAGCATTGA-3'

UFM1 Forward primer: 5'-GTCCCC AGCACACTAGAGGA-3'

Reverse primer: 5'-GGA AAAGAGCGGGAG AGAGT-3'

GAPDH Forward primer: 5'-GAAGGTGAAGGTCGG AGT-3',

Reverse primer: 5'-GAAGATGGTGATGGGATTTC-3'

GAPDH was used as an internal reference, and the $\Delta \Delta \mathrm{Ct}$ method was used for analysis.

\section{Co-immunoprecipitation (Co-IP)}

Protein was extracted from stably transfected cells (HGC-27) overexpressing UFM1, and the BCA method was used to determine the protein concentration. A small amount of protein solution was saved and boiled with $2 \times$ SDS sample buffer and then frozen at $-20^{\circ} \mathrm{C}$ for Western blot analysis. Next, an appropriate amount of UFM1 antibody was added to the remaining protein solution at a ratio of $100 \mu \mathrm{g}$ of protein/1 
$\mu \mathrm{g}$ antibody and incubated at $4^{\circ} \mathrm{C}$ with gentle shaking overnight. Protein $\mathrm{A} / \mathrm{G}$ agarose beads $(20 \mu \mathrm{l})$ were incubated at $4^{\circ} \mathrm{C}$ for $2-4 \mathrm{~h}$ and centrifuged at $4^{\circ} \mathrm{C}$ at $3000 \times \mathrm{g}$ for $3 \mathrm{~min}$. It discarded the supernatant and washed the agarose beads on 5 times with a buffer of $1 \mathrm{ml}$ lysis. After the final removal of the supernatant, $20 \mu \mathrm{l}$ of $2 \times$ SDS was added to the pellet, followed by boiling in water for $5 \mathrm{~min}$. Finally, the CDK5RAP3 antibody was used for Western blot.

\section{Follow-up}

According to the institutional follow-up protocol, qualified doctors monitored all patients by outpatient clinics, phone calls, emails, letters or visits. The first 2 years of follow-up were completed every 3 months. The next 3 years of follow-up were completed every 6 months. Then they were followed up annually until death or after 5 years. Most of the patients had undergone physical exams, laboratory tests, imageological examinations and annual gastroscopy. The time from operation to last follow-up or death was defined as the overall survival time. The follow-up rate of the whole group was $93.56 \%$, and the median follow-up time was 57 months (range, 2-83 months).

\section{Statistical analysis}

All statistical analyses were performed using the Social Science Statistical Software Package (SPSS) version 23.0 for Windows (IBM, Chicago, IL, USA) or R software (version 3.6.2). If not specified, the results were shown as percentages or means $\pm \mathrm{SD}$. As needed, the data were analysed by chi-square test, Fisher's exact test or Student's $t$ test. The survival rate was evaluated by Kaplan-Meier method and log-rank test. The Cox proportional hazards model was used for univariate and multivariate prognostic analysis. Multivariate analysis was performed on factors with $p<0.05$ in univariate analysis. Statistical significance was indicated when the $P$ value was less than 0.05. Pearson's correlation or Spearman's correlation was used to estimate the correlation coefficient $(p<0.05)$. Additionally, the protein interaction network was constructed using GeneMANIA (http://www.genemania.org/). A receiver operating characteristic (ROC) curve and the area under the curve (AUC) were computed to assess discriminative ability.

\section{Results}

\section{The CDK5RAP3 and UFM1 genes were co-downregulated in patients with a poor prognosis}

First, we used unsupervised clustering methods to classify 375 tumour samples from The Cancer Genome Atlas (TCGA) database into three molecular subgroups (Cluster A, Cluster B, and Cluster C) based on the three characteristic pathways of GFRs: KRAS, AKT, and MTOR. The heat map showed that the downstream signalling pathway-related genes GFR signature, GF and GFR were inhibited in patients in Cluster A, while they were activated in patients in Cluster C (Figure 1A). By analysing the related proteins of the GFR pathway from The Cancer Proteome Atlas (TCPA) database, we observed that the GFR pathwayrelated proteins SYK, PDK1, P90RSK, 4EBP1, and BIM were found to be highly expressed in Cluster A, PREX was found to be highly expressed in Cluster B, and CKIT, AMPKALPHA, PKCALPHA_pS657, BAD_pS112, PKCALPHA, PACDELTA_pS664, SHP2542, TUBERIN_pT1462, and IRS1 were found to 
be highly expressed in Cluster C, with significant differences (Figure 1B). Survival analysis also indicated that the overall survival of the patients from Cluster $C$ was lower than that of the patients from Cluster $A$ ( $p$ $=0.043$ ) (Figure 1C). To explore which genes played a key regulatory role in the GFR pathway, we compared the genetic changes in patients in Clusters $B$ vs. $A, C$ vs. $A$, and $C$ vs. B. The Venn diagram showed that Clusters B vs. A, C vs. A and C vs. B had 507 common downregulated genes (Figure 1D and Table S1), and 1,536 common upregulated genes (Figure 1E and Table S2). Analysing the codownregulated genes and CDK5RAP3-interacting proteins in the string database, we found that the CDK5RAP3 and UFM1 genes were included in the 507 common downregulated genes, and the mRNA levels of CDK5RAP3 and UFM1 in patients of category $C$ were lower than those in patients of categories $A$ and $B$. The log fold-change of CDK5RAP3 was -0.741 in Cluster $C$ vs. Cluster A and -0.567 in Cluster $C$ vs. Cluster B. The log fold-change of UFM1 was -0.636 in Cluster C vs. Cluster A and -0.423 in Cluster $C$ vs. Cluster B. (Figure 1F and 1G). Additionally, an interaction was observed between the two proteins (Figure $1 \mathrm{H})$.

\section{UFM1 and CDK5RAP3 were adversely linked to the AKT path activation}

We further performed pathway enrichment analysis of patients with high and low CDK5RAP3 expression in the TCGA and GEO databases. The mountain map, heat map and GSEA enrichment analysis map all indicated that CDK5RAP3 expression negatively correlated with AKT pathway activation (Figures $2 \mathrm{~A}-2 \mathrm{C}$ ), a finding that was consistent with previous research results [6]. Additionally, the correlation analysis of four GEO databases (GSE13861, GSE27342, GSE29272, GSE63089) and the TCGA database revealed that the expression levels of UFM1 and CDK5RAP3 were significantly correlated (Figures 2D-2H). Co-IP experiments confirmed that UFM1 had a direct binding effect with CDK5RAP3 (Figure 2I). Therefore, we knocked down and overexpressed UMF1 in the HGC cell line to verify that UFM1 and CDK5RAP3 negatively correlated with AKT pathway activation. The results showed that knocking down UFM1 caused

a decrease in CDK5RAP3 expression and reduced the inhibition of AKT phosphorylation, while the overexpression of UFM1 caused an increase in CDK5RAP3 to enhance the inhibition of AKT phosphorylation (Figure 2J).

\section{The CDK5RAP3 and UFM1 expression was low in gastric cancer tissue}

Analysis of 7 GEO databases (GSE13861, GSE54129, GSE19826, GSE79973, GSE13911, GSE51575, GSE29272) showed that CDK5RAP3 expression was low in gastric cancer (Figure 3A). CDK5RAP3 expression levels in cancerous and paracancerous tissues from 15 cases in GSE118916, 80 cases in GSE122401, and 47 cases in GSE130823 were found to be low (Figure S2), as was UFM1 expression in cancerous and paracancerous tissues from 15 patients in GSE118916 (Figure 3B). Furthermore, we used samples from the internal centre for verification. IHC staining was used to analyse CDK5RAP3 and UFM1 protein expression in paraffin-embedded gastric cancer samples from 124 patients. CDK5RAP3 was found to be lowly expressed in 102 patients (82.3\%) and had high expressions in 22 patients (17.7\%). The expression levels of UFM1 were found to be low in 93 patients (75.5\%) and high in 31 patients $(25.0 \%)$. Spearman's correlation analysis indicated that CDK5RAP3 and UFM1 expression was significantly correlated (Figure 3C). We also used Western blotting to detect CD K5RAP3 and UFM1 expression in the 
cancerous and paracancerous tissues of 43 gastric cancer patients (Figure 3D) and simultaneously detected the mRNA levels of CDK5RAP3 and UFM1 in the tumour tissues of 48 patients with gastric cancer. Pearson's correlation analysis showed that the expression of the two mRNA levels was positively correlated (Figure 3E).

\section{Patients with low CDK5RAP3 and UFM1 expression had the worst prognosis}

The overall survival was reduced dramatically in patients with low CDK5RAP3 expression compared with patients with high CDK5RAP3 in the 3 GEO databases (GSE13861, GSE15459 and GSE66229) and the TCGA database (Figure S3). Similarly, the overall survival rate was significantly worse among patients with low UFM1 than in patients with high UFM1 (Figure S4). In the GSE66229 database, the patients with low CDK5RAP3 expression had a significant lower disease-free survival rate than that of patients with high CDK5RAP3 expression, and the patients with low UFM1 expression also had a significant lower diseasefree survival rate than those with high UFM1 expression (Figure S5). Regarding the internal centre data, the 3-year overall survival rate was $66.9 \%$ with median 57 months follow-up for the entire group. According to survival analyses, the 3-year cumulative overall survival rate of high CDK5RAP3 expression patients was significantly higher than that of low CDK5RAP3 patients $(81.8 \%$ vs. $62.7 \%, p<0.05$, Figure $4 A)$; those with low UFM1 expression exhibited a lower 3-year overall survival rate than patients with high UFM1 expression ( $58.1 \%$ and $90.3 \%$, respectively; p 0.05 ; Figure 4B). We further analysed the prognostic value of the combination of CDK5RAP3 expression and UFM1 expression by Kaplan-Meier analysis. In comparison to the other groups of patients, patients with low expression levels of CDK5RAP3 and UFM1 had a poorer 3-year cumulative survival rate-only $54.9 \%-$ which was substantially below CDK5RAP3 high and/or UFM1 high expression patients (Figure 4C). After combing the groups, we found that patients with low CDK5RAP3 and UFM1 expression had a significantly worse prognosis than those with high CDK5RAP3 and/or UFM1 expression (88.1\%) $(p<0.001$; Figure 4D).

\section{TNM staging was an independent factor related to the low CDK5RAP3 and UFM1 expression}

Analysis of factors associated with the expression of CDK5RAP3 and UFM1 in gastric cancer tissues showed that the CDK5RAP3 and UFM1 expression significantly correlated with BMI, lymph node metastasis, depth of invasion and pathological TNM stage (Table 1). Combining the low CDK5RAP3 and UFM1 expression, analysis of related factors showed that the low expression level of the two was related to tumour size, depth of invasion, lymph node metastasis and TNM staging (Table 2). BMI, tumour size and TNM staging were further included in the logistic regression model. The results of multivariate analysis suggested that TNM staging was an independent factor related to the low expression of CDK5RAP3 and UFM1 (I+II vs. III: 95\% Cl: $1.128-5.755, p=0.023$ ).

\section{The coexpression level of CDK5RAP3 and UFM1 was an independent prognostic factor for gastric cancer}

Cox regression analyses were used to clarify the prognostic value of CDK5RAP3 and UFM1 expression. Based on the univariate analysis, overall survival was related to BMI, tumour size, TNM staging and combined CDK5RAP3 and UFM1 expression (Table 3). Multivariate analysis indicated that the 
coexpression level of CDK5RAP3 and UFM1, as well as TNM stage were both independent predictive variables for patient prognosis with gastric cancer (Table 3 ).

\section{Combined expression of CDK5RAP3 and UFM1 to improve the accuracy of long-term prognosis evaluation}

We compared the accuracy of CDK5RAP3 or UFM1 expression, as well as combined CDK5RAP3 and UFM1 expression and TNM staging, in predicting gastric cancer survival using ROC curve analysis. The combination expression of CDK5RAP3 and UFM1 was more accurate in predicting patient survival than either CDK5RAP3 or UFM1 expression on its own (AUC was $0.638,0.584$, and $0.596 ; 95 \% \mathrm{Cl}$ was $0.532-$ $0.740,0.473-0.688$, and $0.490-0.702 ; p=0.021,0.172$, and 0.104 for CDK5RAP3 + UFM1, CDK5RAP3 and UFM1 respectively). Additionally, combined CDK5RAP3 and UFM1 expression had a prognostic value that was similar to TNM staging (AUC: $0.651,95 \% \mathrm{Cl}: 0.601-0.786, p=0.001$; Figure $5 \mathrm{~A}$ ). Furthermore, compared with CDK5RAP3 or UFM1 combined with or without TNM staging, the coexpression of CDK5RAP3 and UFM1 combined with TNM staging further improved the prognostic prediction accuracy of patients $(p<0.001$, Figure 5B). Thus, the combination of CDK5RAP3 and UFM1 expression had a higher prognostic ability for overall survival in GC patients.

\section{Discussion}

Gastric cancer remains the third leading cause of death in China despite improvements in diagnosis and therapy in recent years $[5,17]$. To better guide diagnosis and therapy, identifying specific biomarkers linked to gastric cancer prognosis may help improve the accuracy of gastric cancerprognostic assessment [1820]. Based on our previous study and an examination of public databases, this study found that the expression of the UFM1 and CDK5RAP3 genes are downregulated synchronously in gastric cancer patients with poor prognosis and that an interaction occurs between the UFM1 and CDK5RAP3 proteins. Therefore, we chose to evaluate UFM1 as a prognostic factor with CDK5RAP3.

GFRs and their abnormal signal transduction are important mechanisms of tumorigenesis and development, and they have become hot topics of research in recent years [10, 21]. Many studies have shown that the abnormal function of growth factors and their receptors is an important cause of tumour occurrence and development. Such growth factor receptors have tyrosine kinase activity and can regulate the activity of downstream signalling pathways through phosphorylation [22, 23]. The PI3K/Akt signalling pathway plays an important antiapoptotic role. Abnormalities in Akt-related signalling pathways are also associated with the occurrence of various tumours $[13,24]$. Therefore, we used public databases to search for proteins related to the GFR signalling pathway and CDK5RAP3 and attempted to identify biological prognostic indicators for gastric cancer. It was suggested that UFM1 was positively correlated with CDK5RAP3 and its low expression was associated with poorer prognosis of gastric cancer. Previous studies have shown that multiple proteins related to CDK5RAP3 and UFM1 and their modification systems (such as UFC1 and UFL1) are closely related $[14,15,25]$. The correlation analysis of multiple public databases in this study also proved that the CDK5RAP3 and UFM1 expression were found to be substantially linked.. 
To date, few studies have investigated the combined expression levels of CDK5RAP3 and UFM1 and its prognostic significance in gastric cancer. Therefore, in patients with gastric cancer, we assessed the relationship between relevant clinicopathological parameters and overall survival by detecting the CDK5RAP3 and UFM1 expression levels. In univariate analysis, low CDK5RAP3 expression was linked to a poor prognosis, and high UFM1 expression was linked to a better survival rate in gastric cancer patients, indicating that both CDK5RAP3 and UFM1 play a tumour suppressor role in gastric cancer. Further analysis of related factors showed that the CDK5RAP3 and UFM1 coexpression was strongly linked to the invasive depth, lymph node metastasis and TNM stage, indicating that the two proteins are closely related to tumour invasion and migration in gastric cancer. Additionally, we found that the functions of CDK5RAP3 and UFM1 in gastric cancer were positively correlated. Patients with low CDK5RAP3 and UFM1 expression had the worst prognosis; if either of the two proteins showed high expression, patient's prognosis was dramatically better. We considered that because CDK5RAP3 and UFM1 both played a role as tumour suppressor proteins, when one of the two proteins was highly expressed, the tumour suppressor effect in gastric cancer results in no difference in survival. When both proteins were expressed at a low level, the inhibition of the tumour was relieved, resulting in the poorest prognosis of all groups. Further analysis showed that the accuracy of prognostic analysis using CDK5RAP3 and UFM1 expression was closer to the accuracy of TNM staging prognostic analysis and higher than that of using CDK5RAP3 or UFM1 expression alone. Therefore, combination of the CDK5RAP3 and UFM1 expression can improve the capacity to forecast the survival outcomes of patients with gastric cancer.

The TNM staging system has been identified as a major prognostic factor for the gastric cancer. It's also a valuable foundation for the formulation of gastric cancer treatment. However, differences in the prognosis of the same stage patients persist. In this study, we combined the coexpression of CDK5RAP3 and UFM1 with TNM staging for prognostic analysis. In comparison to the conventional TNM staging's forecast accuracy, combining CDK5RAP3 and UFM1 expression with TNM greatly improved the accuracy of predicting gastric cancer patient survival. This finding indicated that the coexpression level of CDK5RAP3 and UFM1 could increase the accuracy of gastric cancer prognostic evaluation. As a result, in clinical practice, the coexpression of CDK5RAP3 and UFM1 can be used in cooperation with TNM staging to effectively guide treatment and follow-up of patients with gastric cancer.

This study mainly explored the impact of the coexpression level of UFM1 and CDK5RAP3 on the clinicopathological parameters of gastric cancer patients and its prognostic significance, providing a preliminary basis for further research. Therefore, the cellular functions of CDK5RAP3 and UFM1 and the elucidation of related mechanisms warrant further investigation.

In summary, we discovered that low CDK5RAP3 and UFM1 was closely linked to the survival outcomes of gastric cancer. Combination of CDK5RAP3 and UFM1 expression was proven to be an independent survival predictor of gastric cancer, resulting in a more accurate prognostic model for the overall survival. Preoperative biopsy and immunohistochemical analysis of CDK5RAP3 and UFM1 protein expression in cancer tissues might help stratify patient treatment algorithms and assess the survival of patients with gastric cancer. Patients with low CDK5RAP3 and/or UFM1, for example, may have a bad prognosis, and 
these patients may require closer follow-up or more aggressive adjuvant treatment after surgery. CDK5RAP3 and UFM1, as effective markers for predicting the outcome of treatment, have considerable clinical and practical value.

\section{Declarations}

\section{Acknowledgments}

We are thankful to Ju-Li Lin, Hua-Long Zheng, Guang-Tan Lin and Fujian Medical University Union Hospital for managing the gastric cancer patient database.

\section{Funding}

This study was funded by Scientific and technological innovation joint capital projects of Fujian Province (No. 2018Y9008). Fujian provincial health technology project (2018-1-40). China scholarship council (202108350068).

\section{Availability of data and materials}

The datasets generated and/or analysed during the current study are partly available in the http://www.ncbi.nlm.nih.gov/geo; https://portal.gdc.cancer.gov/; https://www.tcpaportal.org/tcpa; and https://www.gsea-msigdb.org/. Part of the data that support the findings of this study are available from Fujian Medical University Union Hospital but restrictions apply to the availability of these data, which were used under license for the current study, and so are not publicly available. Data are however available from the authors upon reasonable request and with permission of Fujian Medical University Union Hospital.

\section{Compliance with Ethical Standards}

\section{Conflict of Interest}

The authors declare that they have no conflict of Interest.

\section{Ethical Approval}

All procedures performed in studies involving human participants were in accordance with the ethical standards of the Fujian Medical University Union Hospital research committee and with the 1964 Helsinki Declaration and its later amendments or comparable ethical standards. The experimental protocol was approved by the Fujian Medical University Union Hospital Ethics Committee.

\section{Informed Consent}

Informed consent was obtained from all individual participants included in the study.

\section{Author contributions}


Lin M, Cao LL and Lin JX conceived of the study; Lin M, Lian NZ, Cao LL and Yoon C conducted the experimen and performed the major analysis; Lin M and Lian NZ prepare the manuscript; Lin JX, Yoon C, Huang CM, Zheng CH, Li P, Xie JW, Wang JB , Lu J, Chen QY, Li YH, Peng ZH, Zhang XY and Mei YX helped

to collect the data and revise the manuscript critically for important intellectual content; All authors read and approved the final manuscript.

\section{References}

1. Bray F, Ferlay J, Soerjomataram I, Siegel RL, Torre LA and Jemal A. Global cancer statistics 2018: GLOBOCAN estimates of incidence and mortality worldwide for 36 cancers in 185 countries. CA: a cancer journal for clinicians. 2018; 68:394-24.

2. Smyth EC, Nilsson M, Grabsch HI, van Grieken NC and Lordick F. Gastric cancer. Lancet. 2020; 396:635-48.

3. Cancer Genome Atlas Research N. Comprehensive molecular characterization of gastric adenocarcinoma. Nature. 2014; 513:202-09.

4. Jiang $\mathrm{H}, \mathrm{Wu} \mathrm{J}, \mathrm{He} \mathrm{C}$, Yang $\mathrm{W}$ and $\mathrm{Li} \mathrm{H}$. Tumor suppressor protein $\mathrm{C} 53$ antagonizes checkpoint kinases to promote cyclin-dependent kinase 1 activation. Cell research. 2009; 19:458-68.

5. Ching YP, Qi Z and Wang JH. Cloning of three novel neuronal Cdk5 activator binding proteins. Gene. 2000; 242:285-94.

6. Zheng CH, Wang JB, Lin MQ, Zhang PY, Liu LC, Lin JX, Lu J, Chen QY, Cao LL, Lin M, Tu RH, Xie JW, Li $P$, et al. CDK5RAP3 suppresses Wnt/beta-catenin signaling by inhibiting AKT phosphorylation in gastric cancer. Journal of experimental \& clinical cancer research : CR. 2018; 37:59.

7. Wang JB, Wang ZW, Li Y, Huang CQ, Zheng CH, Li P, Xie JW, Lin JX, Lu J, Chen QY, Cao LL, Lin M, Tu $\mathrm{RH}$, et al. CDK5RAP3 acts as a tumor suppressor in gastric cancer through inhibition of beta-catenin signaling. Cancer letters. 2017; 385:188-97.

8. Hertel P, Daniel J, Stegehake D, Vaupel H, Kailayangiri S, Gruel C, Woltersdorf C and Liebau E. The ubiquitin-fold modifier 1 (Ufm1) cascade of Caenorhabditis elegans. The Journal of biological chemistry. 2013; 288:10661-71.

9. Sasakawa H, Sakata E, Yamaguchi Y, Komatsu M, Tatsumi K, Kominami E, Tanaka K and Kato K. Solution structure and dynamics of Ufm1, a ubiquitin-fold modifier 1. Biochemical and biophysical research communications. 2006; 343:21-26.

10. Ha BH, Jeon YJ, Shin SC, Tatsumi K, Komatsu M, Tanaka K, Watson CM, Wallis G, Chung CH and Kim EE. Structure of ubiquitin-fold modifier 1-specific protease UfSP2. The Journal of biological chemistry. $2011 ; 286: 10248-57$.

11. Yoo HM, Park JH, Jeon YJ and Chung $\mathrm{CH}$. Ubiquitin-fold modifier 1 acts as a positive regulator of breast cancer. Frontiers in endocrinology. 2015; 6:36.

12. Lin JX, Xie XS, Weng XF, Qiu SL, Yoon C, Lian NZ, Xie JW, Wang JB, Lu J, Chen QY, Cao LL, Lin M, Tu $\mathrm{RH}$, et al. UFM1 suppresses invasive activities of gastric cancer cells by attenuating the expres7sion 
of PDK1 through PI3K/AKT signaling. Journal of experimental \& clinical cancer research : CR. 2019; 38:410.

13. Lin J, Cao S, Wang Y, Hu Y, Liu H, Li J, Chen J, Li P, Liu J, Wang Q and Zheng L. Long non-coding RNA UBE2CP3 enhances HCC cell secretion of VEGFA and promotes angiogenesis by activating ERK1/2/HIF-1alpha/VEGFA signalling in hepatocellular carcinoma. Journal of experimental \& clinical cancer research : CR. 2018; 37:113.

14. Kerbel RS. Tumor angiogenesis. The New England journal of medicine. 2008; 358:2039-2049.

15. Loizzi V, Del Vecchio V, Gargano G, et al. Biological Pathways Involved in Tumor Angiogenesis and Bevacizumab Based Anti-Angiogenic Therapy with Special References to Ovarian Cancer. Int J Mol Sci. 2017;18(9):1967.

16. Hoshino Y, Hayashida T, Hirata A, Takahashi H, Chiba N, Ohmura M, Wakui M, Jinno H, Hasegawa H, Maheswaran S, Suematsu M and Kitagawa Y. Bevacizumab terminates homeobox B9-induced tumor proliferation by silencing microenvironmental communication. Molecular cancer. 2014; 13:102.

17. Orditura M, Galizia G, Sforza V, Gambardella V, Fabozzi A, Laterza MM, Andreozzi F, Ventriglia J, Savastano B, Mabilia A, Lieto E, Ciardiello F and De Vita F. Treatment of gastric cancer. World journal of gastroenterology. 2014; 20:1635-49.

18. Roukos $\mathrm{DH}$. Current status and future perspectives in gastric cancer management. Cancer treatment reviews. 2000; 26:243-55.

19. Bittoni A, Maccaroni E, Scartozzi M, Berardi R and Cascinu S. Chemotherapy for locally advanced and metastatic gastric cancer: state of the art and future perspectives. European review for medical and pharmacological sciences. 2010; 14:309-14.

20. Yasui W, Sentani K, Sakamoto N, Anami K, Naito Y and Oue N. Molecular pathology of gastric cancer: research and practice. Pathology, research and practice. 2011; 207:608-12.

21. Fischer KR, Durrans A, Lee S, Sheng J, Li F, Wong ST, Choi H, El Rayes T, Ryu S, Troeger J, Schwabe RF, Vahdat LT, Altorki NK, et al. Epithelial-to-mesenchymal transition is not required for lung metastasis but contributes to chemoresistance. Nature. 2015; 527:472-76.

22. Lamouille S, Xu J and Derynck R. Molecular mechanisms of epithelial-mesenchymal transition. Nature reviews Molecular cell biology. 2014; 15:178-96.

23. Saunders LR and McClay DR. Sub-circuits of a gene regulatory network control a developmental epithelial-mesenchymal transition. Development. 2014; 141:1503-13.

24. Valcourt U, Carthy J, Okita Y, Alcaraz L, Kato M, Thuault S, Bartholin L and Moustakas A. Analysis of Epithelial-Mesenchymal Transition Induced by Transforming Growth Factor beta. Methods in molecular biology. 2016; 1344:147-81.

25. Biondi RM. Phosphoinositide-dependent protein kinase 1, a sensor of protein conformation. Trends in biochemical sciences. 2004; 29:136-42.

\section{Tables}


Table 1. The association of CDK5RAP3 and UFM1 expression in gastric cancer tissues with clinicopathological factors 


\begin{tabular}{|c|c|c|c|c|c|c|c|c|c|}
\hline \multirow[t]{2}{*}{ variables } & \multirow[t]{2}{*}{ Total } & \multicolumn{4}{|c|}{ CDK5RAP3 expression } & \multicolumn{3}{|c|}{ UFM1 expression } & \multirow[b]{2}{*}{ p } \\
\hline & & low & high & $x 2$ & $\mathrm{p}$ & low & high & $x 2$ & \\
\hline Gender & & & & 0.076 & 0.783 & & & 0.341 & 0.5509 \\
\hline Male & 98 & 79 & 19 & & & 73 & 25 & & \\
\hline Female & 26 & 23 & 3 & & & 20 & 6 & & \\
\hline Age (years) & & & & 0.241 & 0.624 & & & 0.168 & 0.682 \\
\hline$>60$ & 87 & 75 & 12 & & & 68 & 19 & & \\
\hline$\leq 60$ & 37 & 27 & 10 & & & 25 & 12 & & \\
\hline $\mathrm{BMI}\left(\mathrm{kg} / \mathrm{m}^{2}\right)$ & & & & 3.910 & 0.048 & & & 4.9119 & 0.027 \\
\hline$>25$ & 23 & 22 & 1 & & & 19 & 4 & & \\
\hline$\leq 25$ & 101 & 80 & 21 & & & 74 & 27 & & \\
\hline $\begin{array}{l}\text { Tumor size } \\
\text { (cm) }\end{array}$ & & & & 0.502 & 0.479 & & & 5.401 & 0.020 \\
\hline$>5$ & 52 & 42 & 10 & & & 34 & 14 & & \\
\hline$\leq 5$ & 78 & 60 & 18 & & & 59 & 17 & & \\
\hline Tumor location & & & & 4.403 & 0.111 & & & 4.191 & 0.123 \\
\hline Lower 1/3 & 55 & 46 & 9 & & & 37 & 18 & & \\
\hline Middle 1/3 & 23 & 19 & 4 & & & 21 & 2 & & \\
\hline Upper 1/3 & 46 & 37 & 9 & & & 35 & 11 & & \\
\hline Borrmann type & & & & 0.209 & 0.647 & & & 0.945 & 0.331 \\
\hline$|+| \mid$ & 27 & 20 & 7 & & & 22 & 5 & & \\
\hline III+IV & 97 & 82 & 15 & & & 71 & 26 & & \\
\hline $\begin{array}{l}\text { Differentiation } \\
\text { type }\end{array}$ & & & & 4.075 & 0.044666 & & & 2.818 & 0.093 \\
\hline Undifferentiated & 78 & 64 & 14 & & & 62 & 16 & & \\
\hline Differentiated & 46 & 38 & 8 & & & 31 & 15 & & \\
\hline pT stage & & & & 8.678 & 0.003 & & & 8.264 & 0.004 \\
\hline $\mathrm{T} 1+\mathrm{T} 2$ & 18 & 13 & 5 & & & 11 & 7 & & \\
\hline T3+ T4 & 106 & 89 & 17 & & & 82 & 24 & & \\
\hline pN stage & & & & 16.478 & 0.001 & & & 19.37774 & 0.000 \\
\hline
\end{tabular}




\begin{tabular}{|c|c|c|c|c|c|c|c|c|c|}
\hline No & 17 & 13 & 4 & & & 11 & 6 & & \\
\hline N1 & 20 & 16 & 4 & & & 15 & 5 & & \\
\hline N2 & 42 & 31 & 11 & & & 32 & 10 & & \\
\hline N3 & 45 & 42 & 3 & & & 35 & 10 & & \\
\hline TNM stage & & & & 11.373 & 0.001 & & & 11.2198 & 0.001 \\
\hline$\nabla+\square$ & 39 & 29 & 10 & & & 26 & 13 & & \\
\hline$\nabla$ & 85 & 73 & 12 & & & 67 & 18 & & \\
\hline Vessel invasion & & & & 0.241 & 0.624 & & & 0.045 & 0.832 \\
\hline Negative & 80 & 62 & 18 & & & 63 & 17 & & \\
\hline Positive & 44 & 40 & 4 & & & 30 & 14 & & \\
\hline
\end{tabular}

Table 2. The association of different CDK5RAP3 and UFM1 expression levels in gastric cancer tissues with clinicopathological factors 


\begin{tabular}{|c|c|c|c|c|c|}
\hline Variables & Total & $\begin{array}{l}\text { CDK5RAP3 and } \\
\text { UFM1 } \\
\text { low expression }\end{array}$ & $\begin{array}{l}\text { CDK5RAP3 and/or UFM1 high } \\
\text { expression }\end{array}$ & $x^{2}$ & p \\
\hline \multicolumn{6}{|l|}{ Gender } \\
\hline Male & 98 & 65 & 33 & 0.462 & 0.497 \\
\hline Female & 26 & 17 & 9 & & \\
\hline \multicolumn{6}{|l|}{ Age (years) } \\
\hline$>60$ & 47 & 21 & 26 & 0.400 & 0.527 \\
\hline$\leq 60$ & 77 & 61 & 16 & & \\
\hline \multicolumn{6}{|l|}{ BMI $\left(\mathrm{kg} / \mathrm{m}^{2}\right)$} \\
\hline$>25$ & 23 & 19 & 4 & 9.666 & 0.002 \\
\hline$\leq 25$ & 101 & 63 & 38 & & \\
\hline \multicolumn{6}{|l|}{$\begin{array}{l}\text { Tumor size } \\
\text { (cm) }\end{array}$} \\
\hline$>5$ & 48 & 31 & 17 & 4.972 & 0.026 \\
\hline$\leq 5$ & 76 & 51 & 25 & & \\
\hline \multicolumn{6}{|l|}{ Tumor location } \\
\hline Lower $1 / 3$ & 55 & 33 & 22 & 3.848 & 0.146 \\
\hline Middle 1/3 & 23 & 18 & 5 & & \\
\hline Upper $1 / 3$ & 46 & 31 & 15 & & \\
\hline \multicolumn{6}{|l|}{ Borrmann type } \\
\hline$|+| \mid$ & 27 & 20 & 7 & 1.146 & 0.284 \\
\hline III+IV & 97 & 62 & 35 & & \\
\hline \multicolumn{6}{|l|}{$\begin{array}{l}\text { Differentiation } \\
\text { type }\end{array}$} \\
\hline Undifferentiated & 78 & 54 & 24 & 3.045 & 0.081 \\
\hline Differentiated & 46 & 28 & 18 & & \\
\hline \multicolumn{6}{|l|}{ pT stage } \\
\hline $\mathrm{T} 1+\mathrm{T} 2$ & 18 & 9 & 9 & 7.965 & 0.005 \\
\hline $\mathrm{T} 3+\mathrm{T} 4$ & 106 & 73 & 33 & & \\
\hline
\end{tabular}




\begin{tabular}{|llllll|}
\hline N0 & 17 & 9 & 8 & 16.236 & 0.001 \\
\hline N1 & 20 & 12 & 8 & & \\
\hline N2 & 42 & 27 & 15 & & \\
\hline N3 & 45 & 34 & 11 & 9.422 & 0.002 \\
\hline TNM stage & & & 19 & & \\
\hline$\square+\square$ & 39 & 20 & 23 & & \\
\hline$\square$ & 85 & 62 & & 0.001 & 0.981 \\
\hline Vessel invasion & & & 26 & & \\
\hline Negative & 80 & 54 & 16 & & \\
\hline Positive & 44 & 28 & & & \\
\hline
\end{tabular}

Table 3. Cox regression analysis of prognostic factor for gastric cancer 
Variable

\begin{tabular}{|c|c|c|c|c|c|c|c|c|}
\hline & & \multirow{2}{*}{\multicolumn{2}{|c|}{$95 \% \mathrm{Cl}$}} & & & \multirow{2}{*}{\multicolumn{2}{|c|}{$95 \% \mathrm{Cl}$}} & \\
\hline & $\mathrm{HR}$ & & & $p$ & $\mathrm{HR}$ & & & \multirow[t]{2}{*}{$\mathrm{p}$} \\
\hline Gender & & & & 0.631 & & & & \\
\hline Female & Ref & & & & & & & \\
\hline Male & 0.849 & 0.43 & 1.65 & 0.631 & & & & \\
\hline Age (years) & & & & 0.960 & & & & \\
\hline$\leq 60$ & Ref & & & & & & & \\
\hline$>60$ & 0.985 & 0.53 & 1.80 & 0.960 & & & & \\
\hline BMI $\left(\mathrm{kg} / \mathrm{m}^{2}\right)$ & & & & 0.020 & & & & 0.156 \\
\hline$\leq 25$ & Ref & & & & Ref & & & \\
\hline$>25$ & 2.078 & 1.12 & 3.85 & 0.020 & 1.579 & 0.84 & 2.97 & 0.156 \\
\hline Tumor size (cm) & & & & 0.044 & & & & 0.069 \\
\hline$\leq 5$ & Ref & & & & Ref & & & \\
\hline$>5$ & 1.758 & 1.02 & 3.05 & 0.044 & 1.685 & 0.96 & 2.95 & 0.069 \\
\hline Tumor location & & & & 0.121 & & & & \\
\hline Lower $1 / 3$ & Ref & & & & & & & \\
\hline Middle 1/3 & 1.246 & 0.56 & 2.78 & 0.590 & & & & \\
\hline Upper $1 / 3$ & 1.879 & 1.01 & 3.47 & 0.044 & & & & \\
\hline Borrmann type & & & & 0.484 & & & & \\
\hline I+ II & Ref & & & & & & & \\
\hline III+IV & 1.293 & 0.63 & 2.66 & 0.484 & & & & \\
\hline Differentiation type & & & & 0.055 & & & & \\
\hline Undifferentiated & Ref & & & & & & & \\
\hline Differentiated & 0.547 & 0.30 & 1.01 & 0.055 & & & & \\
\hline TNM stage & & & & 0.001 & & & & 0.003 \\
\hline$\nabla+\square$ & Ref & & & & Ref & & & \\
\hline$\square$ & 3.885 & 1.75 & 8.65 & 0.001 & 3.320 & 1.48 & 7.42 & 0.003 \\
\hline Vessel invasion & & & & 0.903 & & & & \\
\hline Negative & Ref & & & & & & & \\
\hline
\end{tabular}




\begin{tabular}{lllllllll}
\hline Positive & 0.965 & 0.54 & 1.71 & 0.903 & & & \\
\hline $\begin{array}{l}\text { CDK5RAP3/UFM1 } \\
\text { expression }\end{array}$ & & & & 0.002 & & & 0.006 \\
\hline $\begin{array}{l}\text { CDK5RAP3 low and UFM1 } \\
\text { low }\end{array}$ & Ref & & & & Ref & & \\
\hline $\begin{array}{l}\text { CDK5RAP3 high and/or } \\
\text { UFM1 high }\end{array}$ & 0.312 & 0.15 & 0.64 & 0.002 & 0.357 & 0.17 & 0.74 & 0.006 \\
Figures & & & & & & & & \\
\end{tabular}



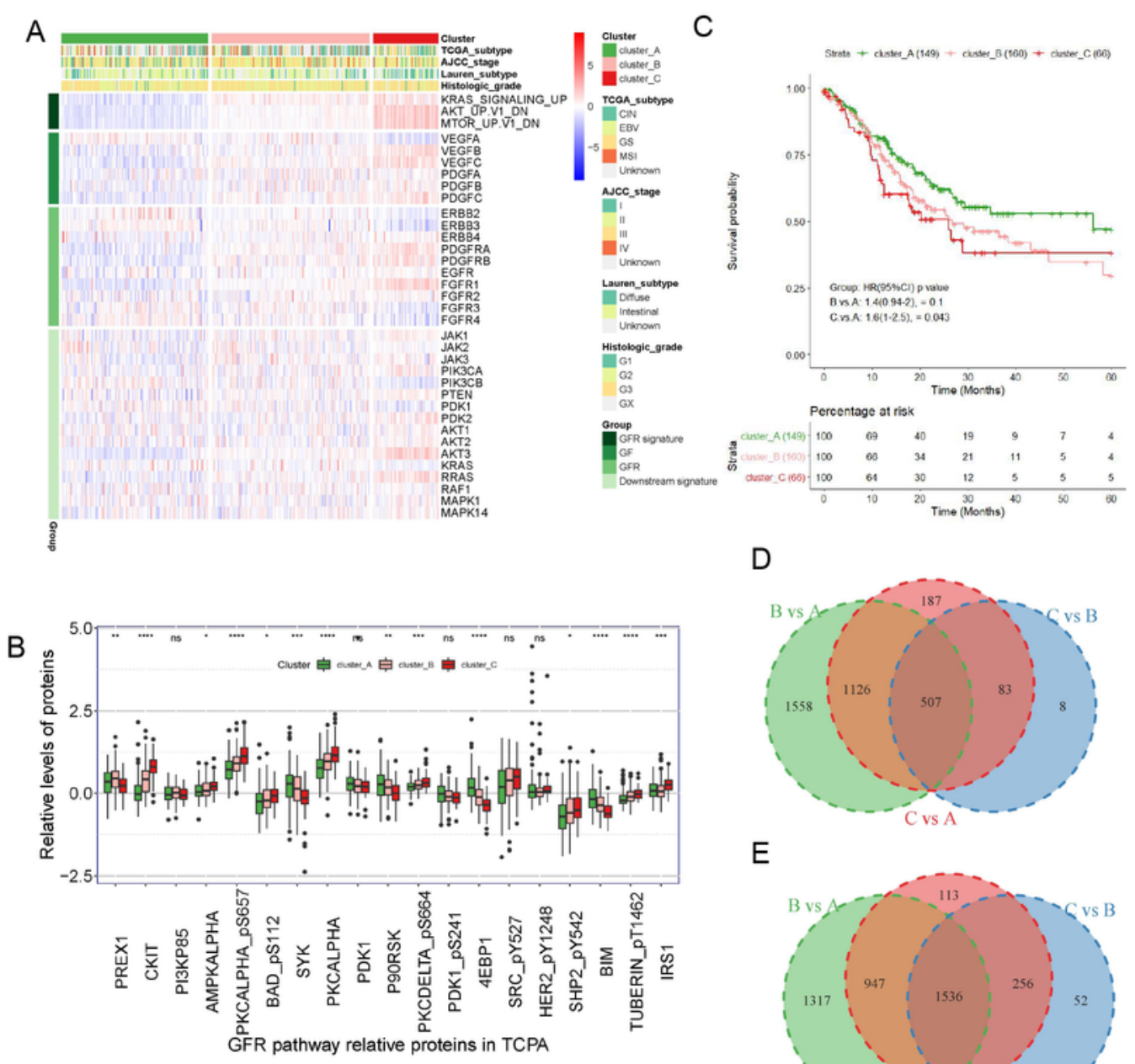

$\mathrm{D}$

$\mathrm{F}$

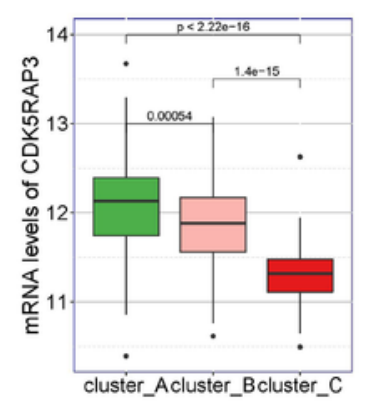

G

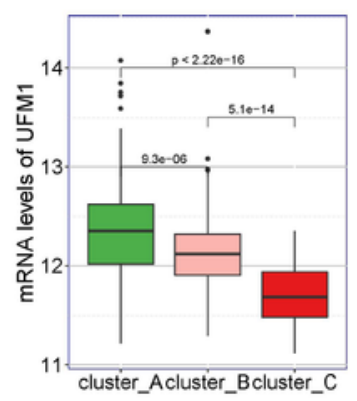

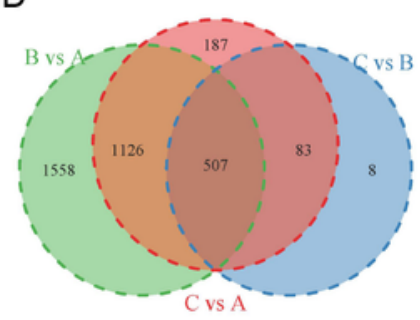

$\mathrm{E}$

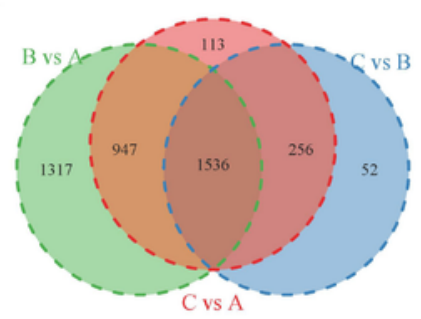

$\mathrm{H}$

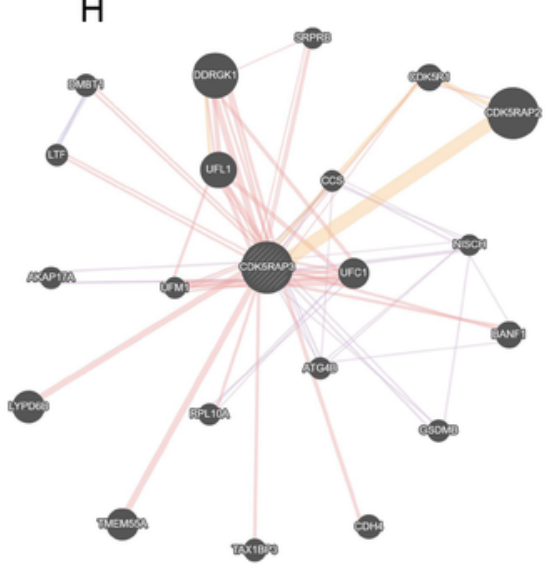

Figure 1

Correlation between UFM1 expression and GFR pathway and CDK5RAP3 expression. (A) Three hundred seventy-five patients in the TCGA cohort were divided into three groups (Cluster A, Cluster B and Cluster C) based on unsupervised analysis and hierarchical clustering of the ssGSEA scores of the three GFR gene sets. (B) Related proteins of the GFR pathway from the TCPA database. (C) Overall survival of patients in Cluster A, Cluster B and Cluster C. (D) Venn diagram of the common downregulated genes in clusters B vs. 
$A, C$ vs. $A$ and $C$ vs. B. (E) Venn diagram of the common upregulated genes in clusters $B$ vs. $A, C$ vs. $A$ and C vs. B. (F) mRNA levels of CDK5RAP3 in the patients in Cluster A, Cluster B and Cluster C. (G) mRNA levels of UMF1 in the patients in Cluster A, Cluster B, and Cluster $\mathrm{C}$. $(\mathrm{H})$ Analysis diagram of the protein interaction network (GeneMANIA).

A

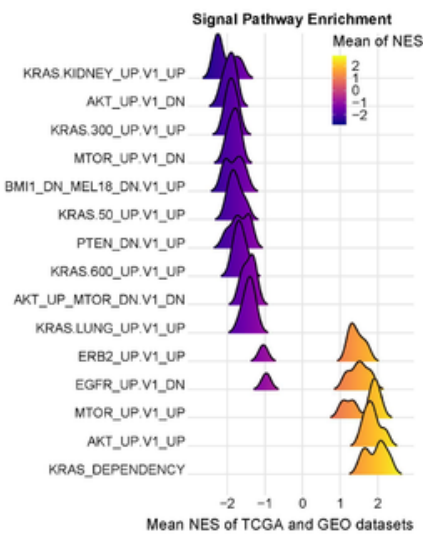

B

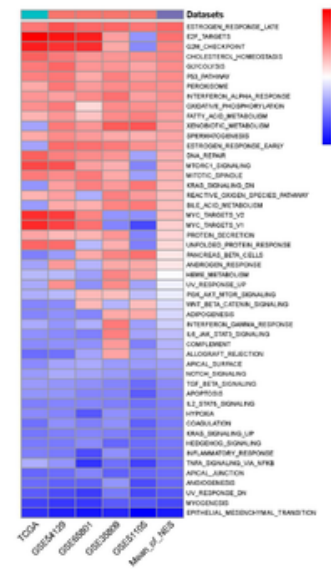

C
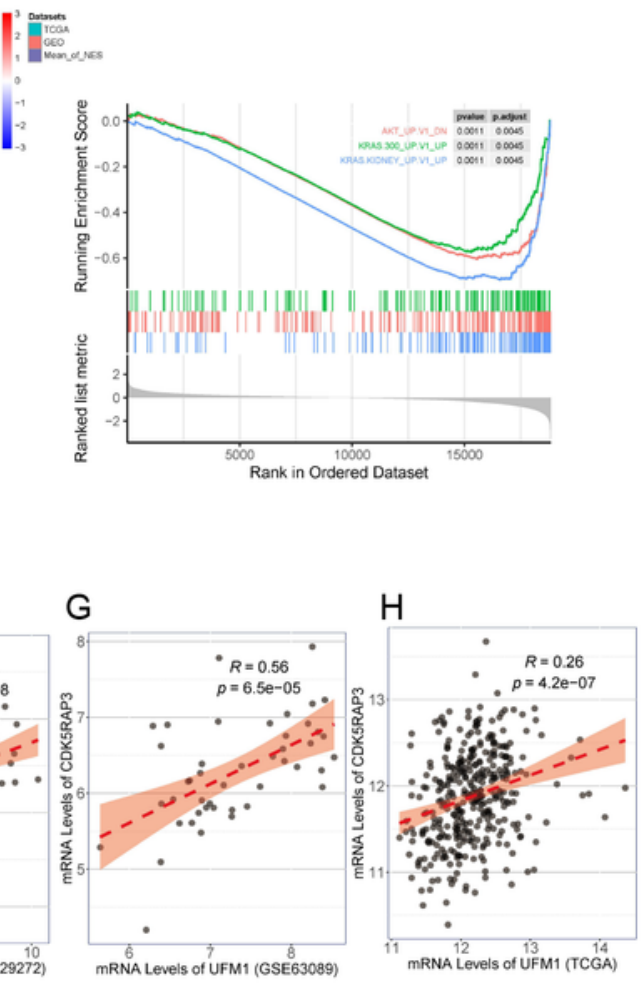

$\mathrm{H}$

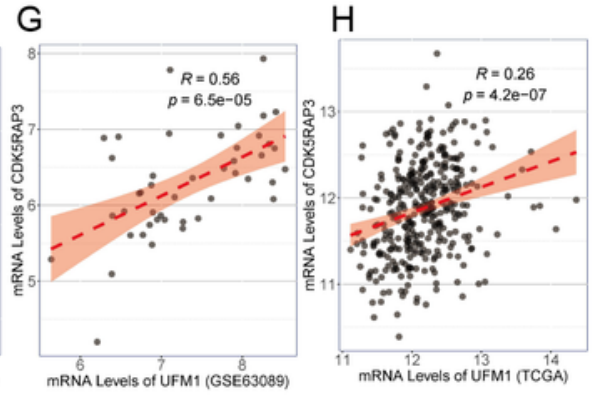

mRNA Levels of UFM1 (GSE63089)

।
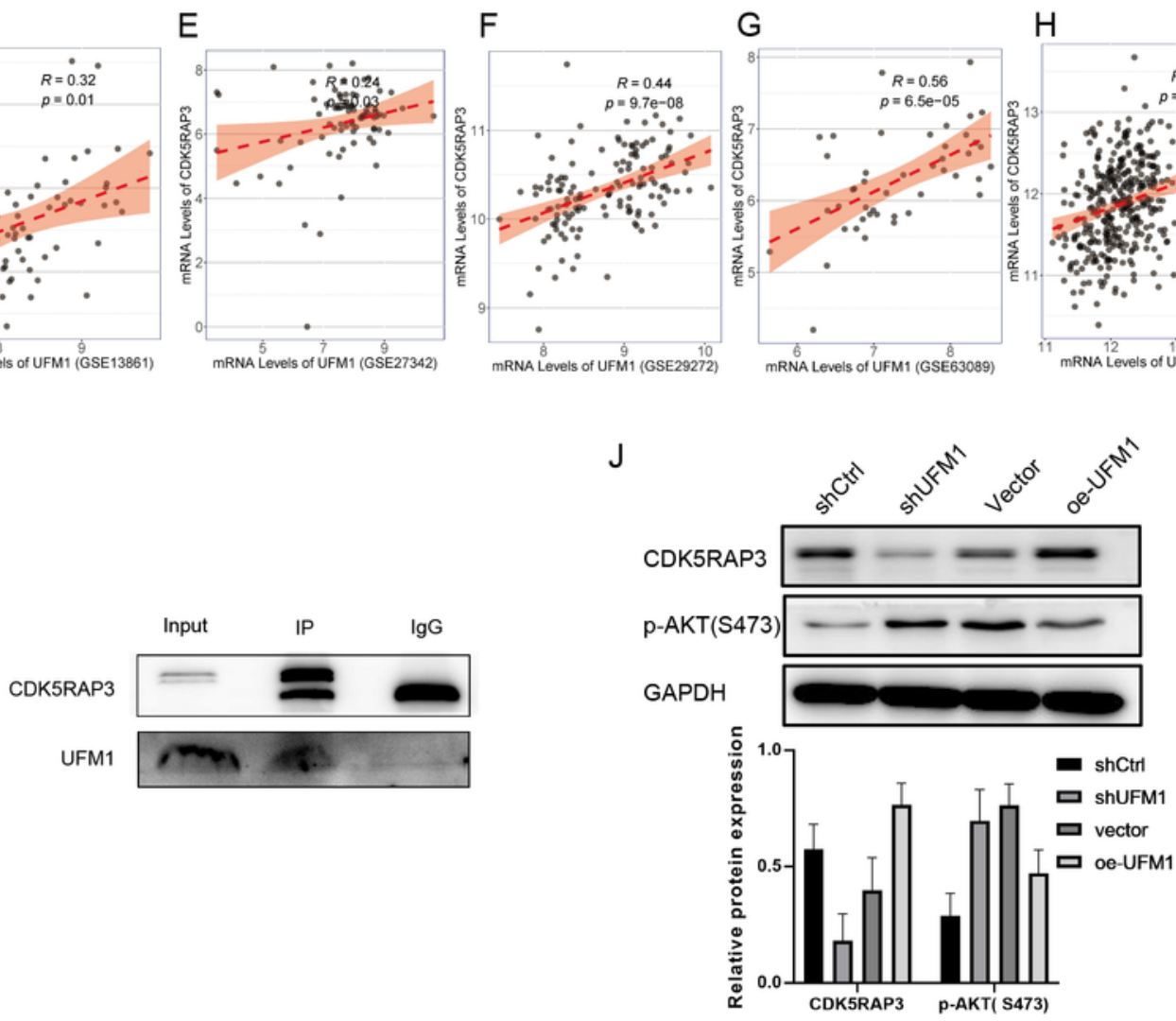

Figure 2 
Pathway enrichment analysis of CDK5RAP3 and the correlation between UFM1 and CDK5RAP3

expression. (A) Mountain map of signal pathway enrichment. (B) Heatmap showing the activation status of the biological processes in different data sets. (C) GSEA enrichment analysis map for CDK5RAP3. (D) Pearson's correlation of UFM1 and CDK5RAP3 expression in GSE13861. (E) Pearson's correlation of UFM1 and CDK5RAP3 expression in GSE27342. (F) Pearson's correlation of UFM1 and CDK5RAP3 expression in GSE29272. (G) Pearson's correlation of UFM1 and CDK5RAP3 expression in GSE63089. (H) Pearson's correlation of UFM1 and CDK5RAP3 expression in TCGA. (I) Co-immunoprecipitation was used to detect the binding of CDK5RAP3 and UFM1 in the overexpressing UFM1 HGC-27 cells. (J) Western blot analysis of CDK5RAP3 and PAKT with knockdown and overexpression of UMF1 in the HGC cell line. 
A

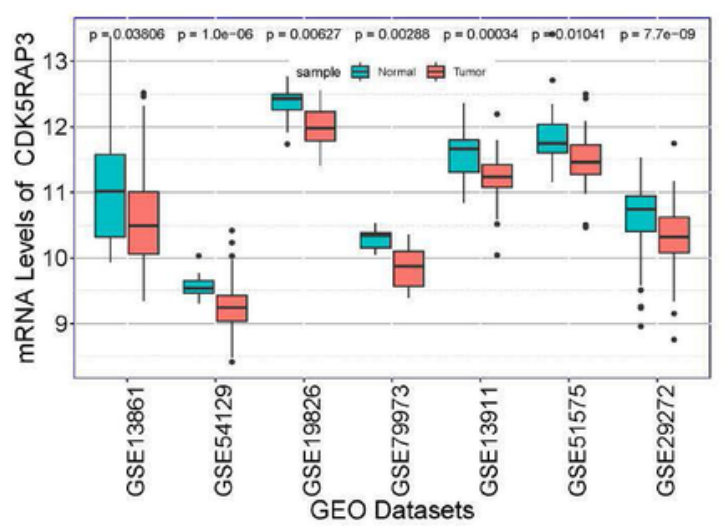

C

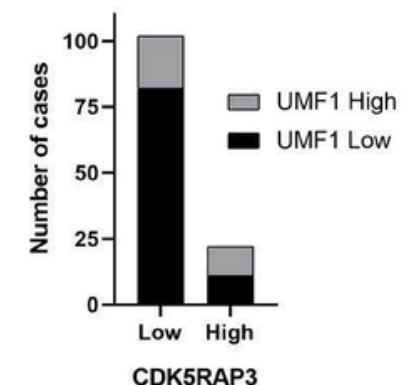

\begin{tabular}{cccc}
\hline \multirow{2}{*}{ UFM1 } & \multicolumn{2}{c}{ CDK5RAP3 } & Spearman $P$ value \\
\cline { 2 - 3 } & Low & High & (correlation coefficient) \\
\hline Low & 82 & 11 & 0.003 \\
\hline
\end{tabular}

$\begin{array}{llll}\text { High } \quad 20 & 11 & 0.268\end{array}$

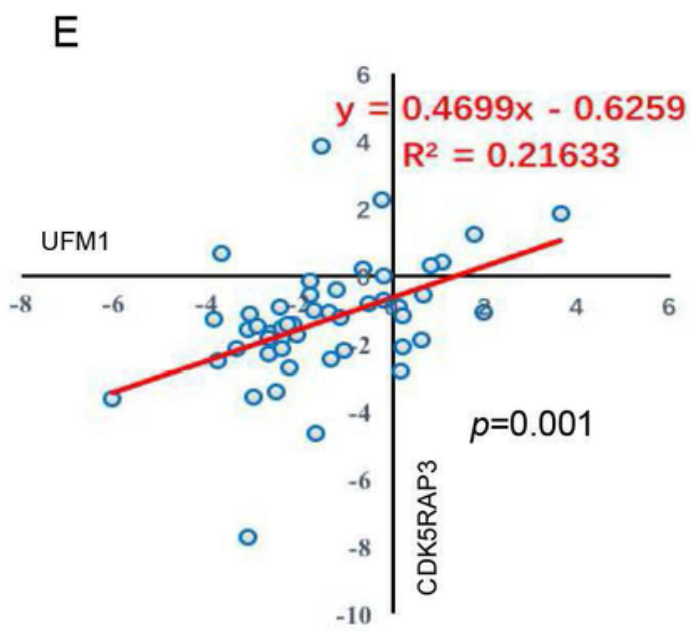

Correlation between UFM1 and CDK5RAP3

mRNA levels
$\mathrm{B}$

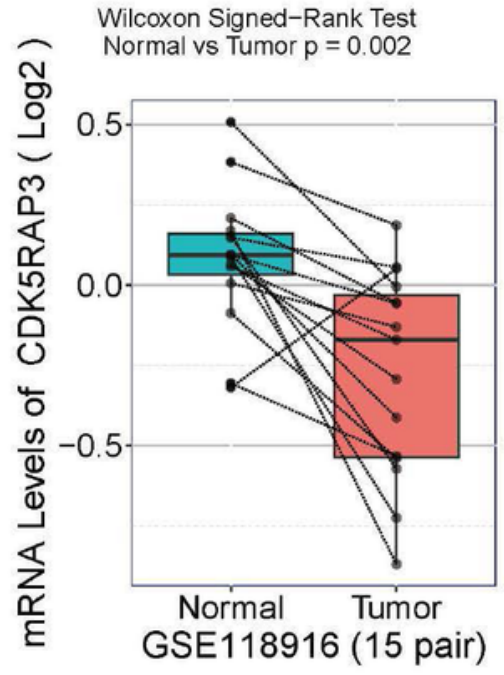

D
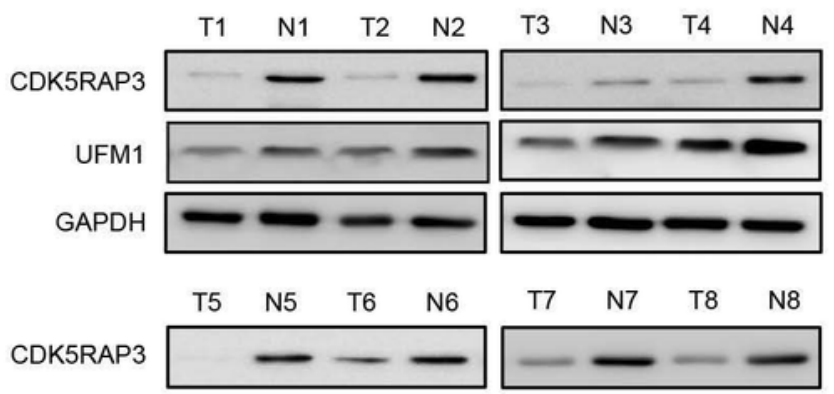

UFM1

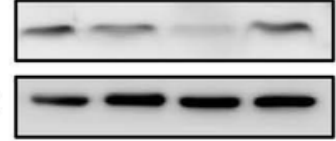

GAPDH $\longrightarrow$

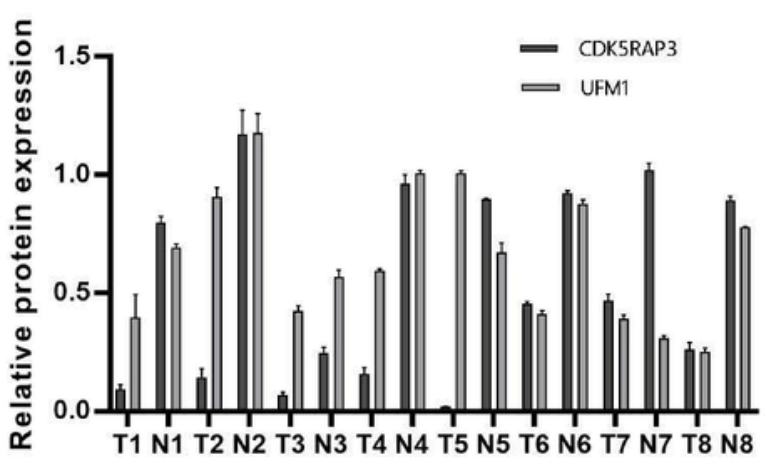

\section{Figure 3}

Expression levels of CDK5RAP3 and UFM1 in gastric cancer tissue. (A) mRNA levels of CDK5RAP3 in cancer and paracancerous tissues in 7 GEO databases. (B) mRNA levels of UMF1 in cancer and paracancerous tissues from 15 cases in GSE118916. (C) CDK5RAP3 was shown to be correlated with UFM1 expression. (D) Protein expression levels of CDK5RAP3 and UFM1 in gastric cancer and 
paracancerous tissues by Western blot. (E) Correlation of CDK5RAP3 and UFM1 mRNA expression in the gastric cancer tissues of 48 patients.

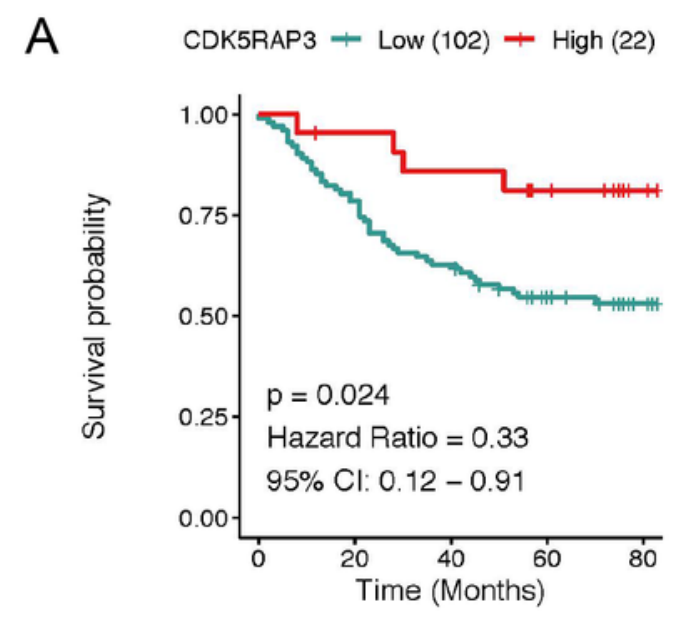

Number at risk
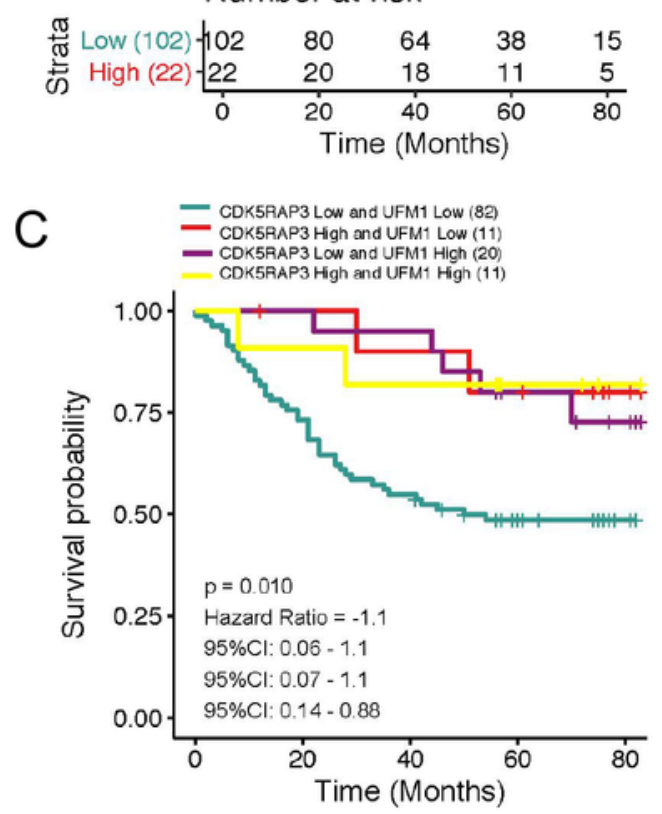

Number at risk

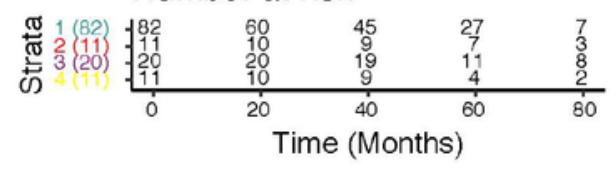

B

UFM1 - Low (93) + High (31)

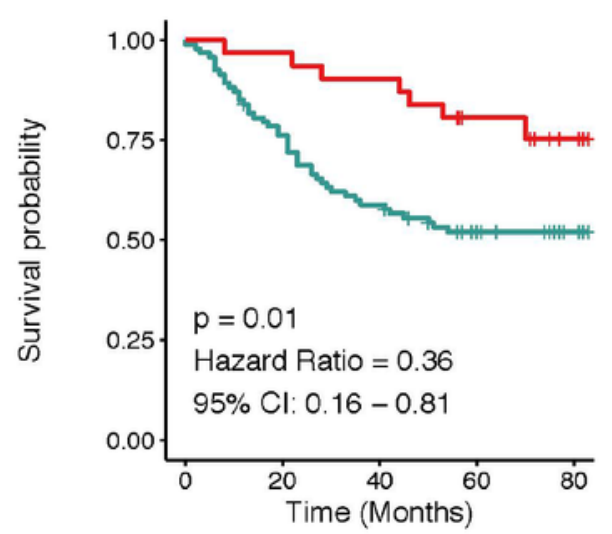

Number at risk

\begin{tabular}{|c|c|c|c|c|c|}
\hline 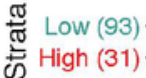 & $\begin{array}{l}93 \\
31\end{array}$ & $\begin{array}{l}70 \\
30 \\
\end{array}$ & $\begin{array}{l}54 \\
28 \\
\end{array}$ & $\begin{array}{l}34 \\
15 \\
\end{array}$ & $\begin{array}{l}10 \\
10\end{array}$ \\
\hline & 0 & 20 & 40 & 60 & 80 \\
\hline
\end{tabular}

D

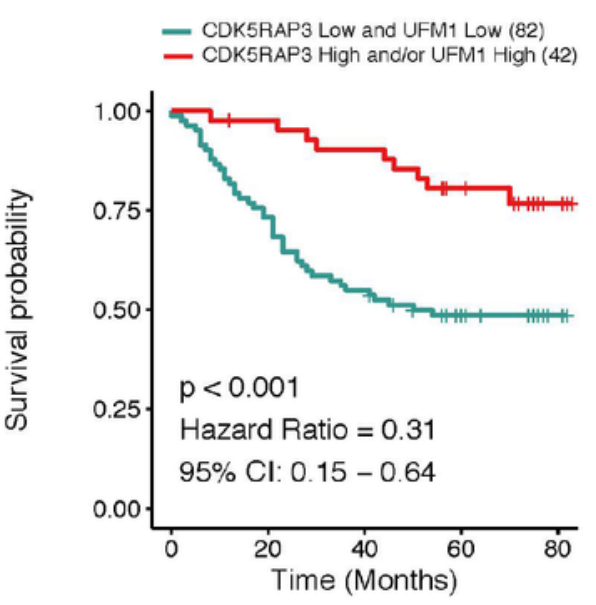

Number at risk

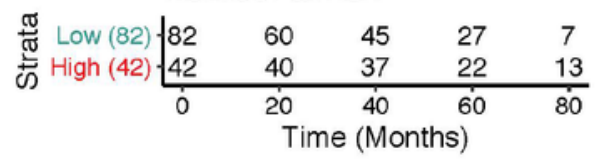

\section{Figure 4}

Overall survival according to different expression levels of CDK5RAP3 and UFM1. (A) Overall survival of gastric cancer patients with low and high expression of CDK5RAP3. (B) Overall survival of gastric cancer 
patients with low and high expression of UFM1. (C) Overall survival of gastric cancer patients with different co-expression of CDK5RAP3 and UFM1. (D) Comparison of the overall survival of CDK5RAP3 low and UFM1 low and CDK5RAP3 high and/or UFM1 high.
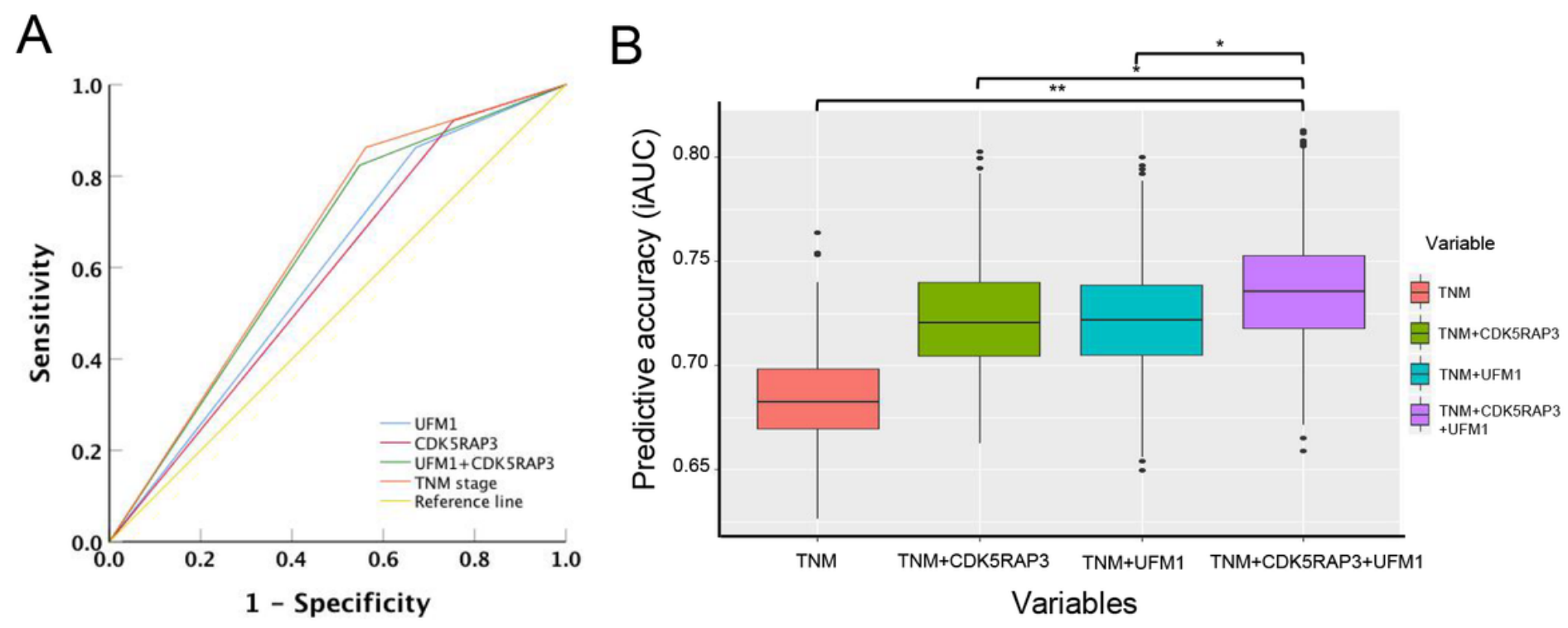

Figure 5

Combined CDK5RAP3 and UFM1 expression was used to evaluate the prognosis of patients with gastric cancer. (A) Receiver operating characteristic analysis of the predictive value. (B) Comparison of the prognostic prediction accuracy of patients among CDK5RAP3 or UFM1 combined with TNM staging, coexpression of CDK5RAP3 and UFM1 combined with TNM staging and separate TNM staging (A box plot depicts the forecast accuracy of the 5-year overall survival based on the iAUC with 1000x bootstrap resampling).

\section{Supplementary Files}

This is a list of supplementary files associated with this preprint. Click to download.

- Supplementaryfiles.docx

- TableS1507commondownregulatedgenes.xls

- TableS21536commonupregulatedgenes.xls 